\title{
Dos décadas de seguimiento exitoso de la primera ablación de arritmias por catéter de radiofrecuencia realizado en la historia de la medicina del Paraguay
}

\section{Osmar Antonio Centurión'}

I. Departamento de Cardiología. Hospital de Clínicas. Universidad Nacional de Asunción. División de Arritmias, Sanatorio Migone-Battilana, Asunción, Paraguay

Cómo referenciar este artículo/ How to reference this article:
Centurión OA. Dos décadas de seguimiento exitoso de la primera ablación de arritmias por catéter de radiofrecuencia realizado en la historia de la medicina del Paraguay. Mem. Inst. Investig. Cienc. Salud, 2015; 13(2): 108-14

\section{R E S U M E N}

La técnica de mapeo endocárdico durante el estudio electrofisiológico ha sido extensamente empleada en el análisis de las taquicardias paroxísticas supraventriculares (TPS). Mediante este mapeo endocárdico y el análisis meticuloso de los electrogramas endocárdicos y los respectivos intervalos de conducción, es posible localizar el sitio de origen de las arritmias y así facilitar su tratamiento ablativo por medio de catéteres de radiofrecuencia. Se describe el caso de un hombre de 29 años con síndrome de WolffParkinson-White (WPW) que manifiestó que presentaba varios episodios documentados de TPS con serio compromiso hemodinámico que motivaba su frecuente internación en terapia intensiva. El mapeo electrofisiológico endocárdico meticuloso demostró la presencia de un haz anómalo de Kent auriculoventricular izquierdo en posición posteroseptal. El período refractario absoluto del haz anómalo de Kent fue de $240 \mathrm{~ms}$. La primera emisión de radiofrecuencia a través de un catéter adecuadamente posicionado previo terminó la taquicardia. La taquicardia permaneció no inducible a partir de entonces. En estas dos décadas de seguimiento clínico, el paciente no ha presentado ni un solo episodio de taquicardia. La curación definitiva generada por la ablación del haz anómalo de Kent ha proporcionado un cambio drástico, total y beneficioso en la calidad de vida al paciente. Los beneficios clínicos y socioeconómicos son mayores cuanto más temprano en la evolución se realice el procedimiento de ablación de arritmias.

Palabras clave: Ablación por radiofrecuencia del haz anómalo de Kent, estudio electrofisiológico, síndrome de Wolff-Parkinson-White, taquicardia paroxística supraventricular.

\section{Two decades of succesful follow-up of the first radiofrequency catheter arrhythmia ablation done in the history of medicine in Paraguay.}

\section{A B S T R A C T}

Endocardial mapping has been widely used for the analysis of supraventricular tachycardias during electrophysiological study. This mapping and the detailed analysis of endocardial electrograms and conduction intervals allow for the localization of the site of origin of the arrhythmias thus facilitating curative treatment with radiofrequency catheter ablation. The case describes our 29 years old patient with manifested Wolff-ParkinsonWhite syndrome that presented frequent, documented episodes of paroxysmal 
supraventricular tachycardia with serious hemodynamic alteration that needed frequent admissions to intensive care units despite the use of 2-3 antiarrhythmic agents per day. A detailed endocardial mapping showed a left posteroseptal accessory pathway. The accessory pathway effective refractory period is $240 \mathrm{~ms}$. The first radiofrequency emission through an adequately positioned radiofrequency catheter terminated the tachycardia. The tachycardia was rendered non-inducible thereafter. In two decades of follow-up, the patient has not presented a single episode of tachycardia. The definite cure provided by the radiofrequency ablation produced a total, dramatic, and beneficial change in the quality of life of the patient. The clinical and socio-economical benefits are greater the earlier the arrhythmia ablation procedure is performed.

Keywords: Radiofrequency ablation of the Kent accessory pathway, electrophysiological study, wolff-Parkinson-White síndrome, paroxysmal supraventricular tachycardia.

\section{NTRODUCCI ÓN}

Mediante el análisis detallado y meticuloso de los electrogramas endocárdicos y de los intervalos de conducción auriculoventricular en forma anterógrada y retrógrada se pueden determinar las características electrofisiológicas del circuito de reentrada, los riesgos de morbilidad y mortalidad, tambien localizar exactamente donde se encuentra el haz anómalo de Kent, conocer sus propiedades eléctricas y además realizar la interrupción definitiva de la conducción a través de sus fibras (1-3). La velocidad de conducción de un haz anómalo auriculoventricular depende de la orientación de las fibras del haz, del período refractario, y de la longitud y geometría de la vía accesoria (4-7). Por lo tanto, existen haces anómalos con conducción muy rápida que tienen un alto riesgo de morbilidad-mortalidad. El peligro aumenta mucho más aún cuando se produce una fibrilación auricular paroxística en un paciente con síndrome de Wolff-Parkinson-White (WPW), portador de un haz anómalo con conducción anterógrada rápida (8-10), ya que pasan muchos impulsos al ventrículo y se puede desencadenar un episodio de fibrilación ventricular y muerte súbita (11-18).

Es nuestra intención reportar en este artículo la primera ablación de arritmias realizada en la historia de la medicina en Paraguay en forma exitosa con un seguimiento a largo plazo de dos décadas en un hombre de 29 años con síndrome de WPW sin cardiopatía orgánica que presentaba varios episodios documentados de taquicardia paroxística supraventricular con serio compromiso hemodinámico y pre-sincope que motivaba su internación frecuente en terapia intensiva, a pesar del uso concomitante de 2-3 fármacos antiarritmicos.

\section{REPORTE DEL CASO}

Un paciente de sexo masculino de 29 años con síndrome de Wolff-Parkinson-White y episodios recurrentes de taquicardia paroxística supraventricular con seria alteración hemodinámica y pre-sincope fue referido al Departamento de Hemodinamia y Electrofisiología del Sanatorio Migone-Battilana para un estudio electrofisiológico y ablación de arritmias por radiofrecuencia. El examen físico, radiológico y los análisis laboratoriales estaban dentro de límites normales. Otros estudios auxiliares de diagnóstico mostraron un electrocardiograma con síndrome de pre-excitación, síndrome de Wolff-Parkinson-White tipo A y una ecocardiografia Doppler que descartaba todo tipo de cardiopatía orgánica. El paciente presentaba varios episodios documentados de taquicardia paroxística supraventricular con compromiso hemodinámico extremo y pre - sincope que motivaba su internación una a dos veces al mes durante varios años, e inclusive en terapia intensiva, a pesar del uso concomitante de 2-3 fármacos antiarrítmicos. 
Estudio Electrofisiológico: El paciente fue estudiado en ayunas, sin sedación, después de haber obtenido el respectivo consentimiento informado. Todos los fármacos fueron suspendidos por lo menos por $72 \mathrm{Hs}$ antes del procedimiento, y por lo menos por un mes en el caso de amiodarona. Cuatro electrodos catéteres cuadripolares ( No6 $6 \mathrm{~F} \mathrm{USCl}$ ) fueron introducidos percutáneamente por técnica Seldinger en la vena femoral derecha y la vena subclavia izquierda y fueron avanzados hacia las cavidades cardiacas bajo observación fluoroscópica. La distancia entre los electrodos del catéter era de $10 \mathrm{~mm}$. y el diámetro del anillo del electrodo era de $2 \mathrm{~mm}$.

Todos los electrogramas auriculares endocardios fueron registrados durante ritmo sinusal mediante catéteres bipolares con ganancia fija y acompañados de una señal de calibración de $0.2 \mathrm{mV}=3 \mathrm{~mm}$. y filtrados a $50-1000 \mathrm{~Hz}$. La linea de base de los registros fue estable. Los electrogramas auriculares y ventriculares endocardios conjuntamente con derivaciones electrocardiográficas ( $\mathrm{I}, \mathrm{aVF}$, y $\mathrm{V} 1$ ) fueron observados en un osciloscopio multicanal (Marquette Electronics Polygraph, Jupiter, USA ) y simultáneamente registrados a una velocidad de papel de $100 \mathrm{~mm} / \mathrm{seg}$ por un Mingograph a 12 canales (Marquette Electronics, Júpiter, USA).

El día 13 de noviembre de 1.995 se realizó una estimulación cardiaca programada con cuatro catéteres electrodos ( $\mathrm{N}^{\circ} 6 \mathrm{~F}, \mathrm{USCl}$, Div. CR. Bard, Billerrica, MA, USA) tripolares y cuadripolares introducidos por abordaje percutáneo modificado de Seldinger a través de las venas femorales y subclavia izquierda. Los catéteres electrodos fueron posicionados en el apéndice auricular derecho, en la punta del ventrículo derecho, a través de la valva septal de la tricúspide y en el seno coronario. No se observo ninguna complicación como resultado de estas maniobras. La posición de la punta de los catéteres fue verificada por fluoroscopia monoplanar.

El mapeo endocárdico meticuloso demostró la presencia de un haz anómalo de Kent auriculoventricular izquierdo en posición posteroseptal. Los intervalos de conducción en ritmo sinusal y los períodos refractarios obtenidos por estimulación programada por extraestimulo simple auricular y ventricular se presentan en la Tabla. La taquicardia paroxística supraventricular de tipo ortodrómica se indujo fácilmente y en forma reproducible mediante la estimulación rápida auricular $\mathrm{y} / \mathrm{o}$ ventricular, así como, con estimulación programada con extraestimulo simple. Se observó un típico aumento de la pre-excitación ventricular con la disminución progresiva del intervalo de acoplamiento del extraestímulo auricular.

El día 28 de noviembre de 1.995 se procedió a la ablación con catéter por radiofrecuencia. A través del catéter cuadripolar de radiofrecuencia (Marinr 7F, Medtronics, USA), se procedió a la emisión de radiofrele.

radiofrecuencia intrataquicardia inducida, terminó la taquicardia y se tornó no inducible. El tiempo de conducción AV a nivel del seno coronario proximal durante ritmo sinusal se prolonga de $70 \mathrm{~ms}$ a $170 \mathrm{~ms}$ (Figuras 1 y 2). Seguidamente se procedió a la emisión de consolidación de radiofrecuencia por espacio de $30 \mathrm{seg}$. y luego ya no se pudo inducir la taquicardia post-ablación. El paciente fue dado de alta antes de las 48 hs sin tratamiento antiarrítmico y actualmente ya lleva dos décadas de seguimiento clínico y no volvió a presentar ni un solo episodio de taquicardia paroxistica supraventricular. 
Tabla 1. Intervalos de conducción en ritmo sinusal y datos electrofisiológicos del sistema de conducción.

\begin{tabular}{ll}
\hline Longitud de ciclo durante ritmo sinusal & $510 \mathrm{~ms}$ \\
Tiempo de conducción intra-auricular & $95 \mathrm{~ms}$ \\
Tiempo de conducción inter-auricular & $75 \mathrm{~ms}$ \\
Tiempo de conducción intra-His y fascicular & $35 \mathrm{~ms}$ \\
Intervalo P-A & $35 \mathrm{~ms}$ \\
Umbral del músculo auricular: & $0.2 \mathrm{mV}$ \\
Conducción AV 1/1 & $230 \mathrm{lat} / \mathrm{min}$ \\
Bloqueo AV 2/1 & $240 \mathrm{lat} / \mathrm{min}$. \\
No se observó bloqueo AV tipo Wenckebach. & - \\
Período refractario auricular absoluto & $190 \mathrm{~ms}$ \\
Período refractario auricular funcional & $280 \mathrm{~ms}$ \\
Umbral del músculo ventricular & $0.5 \mathrm{mV}$ \\
Conducción VA 1/1 & $250 \mathrm{lat} / \mathrm{min}$ \\
Conducción VA 2/1 & $>250 \mathrm{lat} / \mathrm{min}$ \\
Período refractario ventricular absoluto & $190 \mathrm{~ms}$ \\
Período refractario ventricular funcional & $220 \mathrm{~ms}$ \\
\hline
\end{tabular}

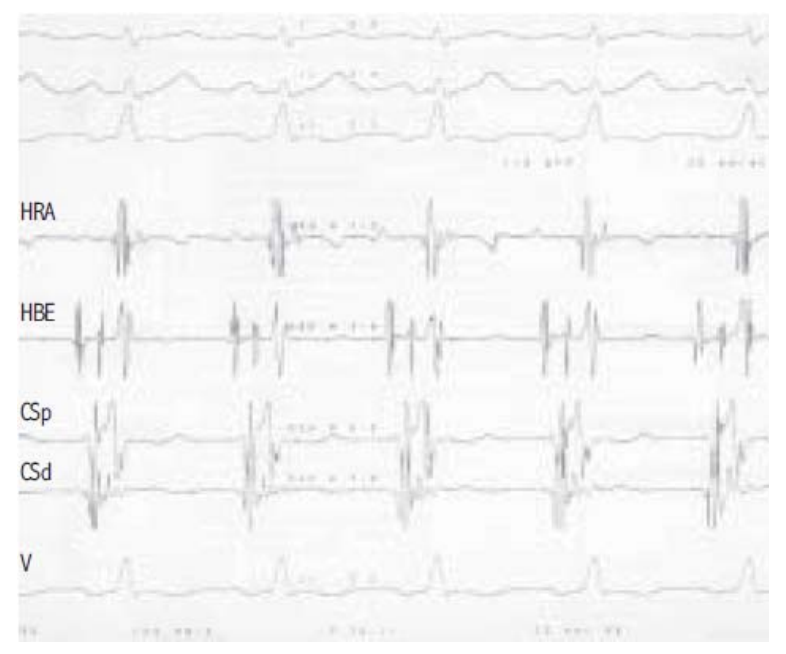

Figura 1. Registro electrofisiológico pre-ablación durante el ritmo sinusal, en el que se observan tres derivaciones electrocardiográficas y los electrogramas endocárdicos de la parte alta de la aurícula derecha (HRA), del haz de His (HBE), seno coronario proximal (CSp), seno coronario distal (SCd) y de la punta del ventrículo derecho (V). El intervalo AV a nivel del seno coronario proximal es de $70 \mathrm{~ms}$. 


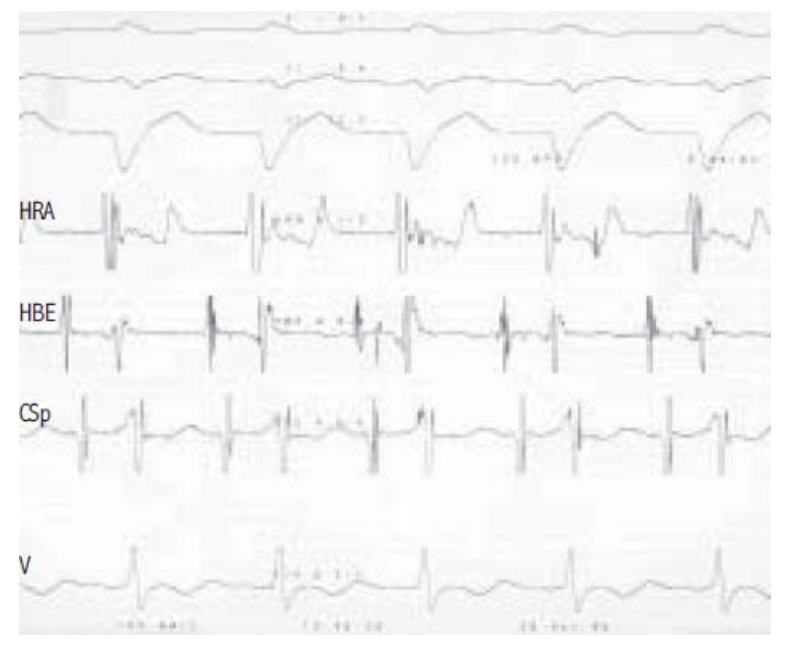

Figura 2. Registro electrofisiológico posablación por radiofrecuencia del haz anómalo de Kent durante el ritmo sinusal, en el que se observan tres derivaciones electrocardiográficas y los electrogramas endocárdicos de la parte alta de la aurícula derecha (HRA), del haz de His (HBE), seno coronario proximal (CSp), y de la punta del ventrículo derecho (V). El intervalo AV a nivel del seno coronario proximal es de $170 \mathrm{~ms}$. Después de la interrupción de la conducción a través del haz anómalo de Kent posablación por radiofrecuencia existe un aumento del tiempo de conducción del intervalo AV a nivel del seno coronario proximal. Se observo un bloqueo transitorio de la rama izquierda, probablemente por edema perilesional.

\section{DISCUSIÓN}

En la práctica clínica, es muy frecuente tratar a los pacientes con TPS con antiarrítmicos en forma empírica. Un número importante de estos pacientes responde favorablemente, generalmente espaciando la frecuencia de aparición de la taquicardia y/o disminuyendo la duración del episodio agudo. Sin embargo, muchos pacientes siguen padeciendo las crisis episódicas de TPS sin mejoría alguna de los síntomas que padecen y alteran de forma muy negativa su calidad de vida. En estos casos, son varios los factores que influyen en la decisión terapéutica. Entre ellos tenemos la preferencia del médico tratante, la tolerabilidad del paciente a las crisis agudas, la condición socioeconómica del paciente en nuestro medio, y las condiciones médicas asociadas. Todos estos factores juegan un papel fundamental en la determinación del tratamiento inicial del paciente. Lastimosamente, la mayoría de las veces la elección del tratamiento inicial de los episodios de TPS que presenta el paciente no se traduce en un tratamiento definitivo, un tratamiento que signifique una curación definitiva de las dolencias que aquejan al paciente $(19,20)$. El tratamiento curativo y definitivo por medio de la ablación con catéter por radiofrecuencia es altamente atractivo, de probado beneficio costo-eficacia, con alta incidencia de seguridad y muy baja tasa de complicaciones, por lo que ha revolucionado el manejo terapéutico de estos pacientes $(21,22)$.

En este artículo se reporta la primera ablación de arritmias realizada en la historia de la medicina del Paraguay en forma exitosa con un seguimiento de dos décadas en un hombre de 29 años con síndrome de WPW sin cardiopatía orgánica que presentaba varios episodios documentados de taquicardia paroxística supraventricular con serio compromiso hemodinámico y pre-sincope que motivaba su internación una a dos veces al mes durante varios años, e inclusive en terapia intensiva, a pesar del uso concomitante de 2-3 
fármacos antiarritmicos. El paciente fue manejado por varios años por internistas y cardiólogos clínicos mediante tratamiento conservador con prácticamente todos los antiarrítmicos disponibles en plaza en nuestro medio. Debido al fracaso del tratamiento médico, el paciente fue referido a nuestro servicio para tratamiento definitivo y curativo con ablación por catéter de radiofrecuencia.

La TPS desencadenada en nuestro paciente utilizaba en su circuito de reentrada al nódulo AV en forma anterógrada y a un haz anómalo de Kent posteroseptal izquierdo en forma retrógrada, con una conducción ventriculo-auricular de $70 \mathrm{~ms}$. La longitud de ciclo de la taquicardia inducida es de $280 \mathrm{~ms}$. El período refractario absoluto del haz anómalo de Kent es de $240 \mathrm{~ms}$. Estos parámetros electrofisiológicos denotan una velocidad de conducción rápida a través del haz de Kent y un periodo refractario corto que lo ubican en la categoría de alto riesgo de mortalidad. Evidentemente esto demuestra la razón por la que el paciente presentaba una alta respuesta ventricular y una seria alteración hemodinámica y pre-síncope.

En conclusión, el análisis detallado y meticuloso de los electrogramas endocárdicos y los intervalos de conducción mediante el estudio electrofisiológico permitió la interrupción exitosa de la conducción a través del haz anómalo de Kent con la primera y única emisión de radiofrecuencia. Es evidente el contraste en la calidad de vida y la evolución clínica en los períodos pre y post-ablación. La curación definitiva generada por la ablación del haz anómalo de Kent ha proporcionado un cambio drástico, total y beneficioso en la calidad de vida al paciente, quién en un período de dos décadas no ha presentado ni un solo episodio de taquicardia paroxística supraventricular. Los efectos positivos de la farmacoterapia en los episodios agudos no necesariamente se traducen en prevención exitosa de las recurrencias. Los cambios en las propiedades electrofisiológicas anterógradas del haz de Kent inducidos por el antiarrítmico no necesariamente son similares a los producidos en su conducción retrógrada. Los beneficios clínicos y socioeconómicos son mayores cuando más temprano en la evolución de la patología se realiza la ablación de la arritmia y por ende, su curación definitiva.

\section{REFERENCI AS BI BLI OGRÁFICAS}

1.Wellens HJ, Durrer D. Patterns of ventriculoatrial conduction in the Wolff-Parkinson-White syndrome. Circulation. 1974; 49(1):22-31.

2.Svenson RH, Miller HC, Gallagher JJ, Wallace AG. Electrophysiological evaluation of the WolffParkinson-White syndrome: Problems in assessing antegrade and retrograde conduction over the accessory pathway. Circulation. 1975; 52(4): 552-62.

3.Narula OS. Retrograde pre-excitation: Comparison of antegrade and retrograde conduction intervals in man. Circulation. 1974; 50(6): 1129-43.

4. Becker AE, Anderson RH, Durrer D, Wellens HJ . The anatomical substrates of Wolff-ParkinsonWhite syndrome: A clinicopathologic correlation in seven patients. Circulation. 1978;57(5):8709.

5.Jackman WM, Friday $\mathrm{KJ}$, Yeung-Lai-Wah JA, Fitzgerald DM, Beck B, Bowman AJ, et al. New catheter technique for recording left free-wall accessory atrioventricular pathway activation: Identification of pathway fiber orientation.
Circulation. 1988; 78(3): 598-611.

6.Critelli G, Gallagher JJ, Monda V, Coltorti F, Scherillo $M$, Rossi L. Anatomic and electrophysiologic substrate of the permanent form of junctional reciprocating tachycardia. J AM Coll Cardiol. 1984; 4 (3): 601-10.

7.De la fuente D, Sasyniuk B, Moe GK. Conduction through a narrow isthmus in isolated canine atrial tissue: A model of the W-P-W syndrome. Circulation 1971; 44 (5) : 803-9.

8. Della Bella $P$, Brugada $P$, Talajic $M$, Lemery $R$, Torner $P$, Lezaun $R$, et al. Atrial fibrillation in patients with an accessory pathway: Importance of the conduction properties of the accessory pathway. J Am Coll Cardiol. 1991; 17(6): 1352-6.

9.Fananapazir L, German LD, Gallagher JJ, Lowe JE, Prystowsky EN. Importance of preexcited QRS morphology during induced atrial fibrillation to the diagnosis and localization of multiple accessory pathways. 
Circulation 1990; 81(2):578-85.

10. Robinson K, Rowland E, Krikler DM. WolffParkinson-White syndrome: Atrial fibrillation as the presenting arrhythmia. Br Heart J. 1988;59 (5): 578-80.

11.Sharma AD, Klein GJ, Guiraudon GM, Milstein S. Atrial fibrillation in patients with WolffParkinson-White syndrome: Incidence after surgical ablation of the accessory pathway. Circulation 1985; 72(1): 161-9.

12. Bauerfeind RA, Wyndham CR, Swiryn SP, Palileo EV, Strasberg B, Lam W, et al. Paroxysmal atrial fibrillation in the WolffParkinson-White syndrome. Am J Cardiol. 1981; 47(3):562-9.

13. Campbell RW, Smith RA, Gallagher JJ, Pritchett EL, Wallace AG. Atrial fibrillation in the pre-excitation syndrome. Am J Cardiol. 1977; 40(4):514-20.

14. Amat-y-Leon F, Dhingra RC, Wu D, Denes P, Wyndham, C, Rosen KM. Catheter mapping of retrograde atrial activation: Observations during ventricular pacing an AV nodal re-entrant paroxysmal tachycardia. $\mathrm{Br}$ Heart J. 1976; 38 (4):355-62.

15. Kastor JA, Goldreyer BN, Moore EN, Shelburne JC, Manchester JH. Intraventricular conduction in man studied with an endocardial electrode catheter mapping technique: Patients with normal QRS and right bundle branch block. Circulation. 1975; 51(5):786-96.

16. Centurión OA, Fukatani M, Shimizu A, Konoe A, I somoto $S$, Tanigawa $M$, et al. Anterograde and retrograde decremental conduction over left -sided accessory atrioventricular pathways in the Wolff-Parkinson- White syndrome. Am Heart J. 1993; 125(4):1038-47.

17. Centurión OA, Isomoto S, Hayano M, Yano K. Evidence of quadruple anterograde atrioventricular nodal pathways in a patient with atrioventricular node reentry. J Electrocardiol. 1994; 27(1): 71-8.

18. Centurión OA, Isomoto S, Konoe A, Shimizu A, Hayano M, Yano K. Electrophysiologic demonstration of anterograde fast and slow pathways within the His bundle in patients with normal intraventricular conduction. Int J Cardiol. 1994; 44(3):251-60.

19. Kaibara M, Konoe A, Isomoto S, Centurión OA, Hirata T, Hano O, et al. Radiofrequency catheter ablation for atrioventricular node reentry tachycardia with multiple slow atrioventricular node pathways. Jap Circulation J. 1995; 59(4):224-30.

20.Centurión OA, Kaibara $M$, Isomoto $S$, Konoe A, Yano K. Unmasking of fast and slow atrioventricular nodal pathways by successful radiofrequency ablation of two accessory atrioventricular connections. Clin Cardiol. 1996; 20(1): 75-8.

21.Jackman WM, Wang $X$, Friday $\mathrm{KJ}$, Roman $\mathrm{CA}$, Moulton KP, Beckman KJ, et al. Catheter ablation of accessory atrioventricular pathways (Wolff-ParkinsonWhite syndrome) by radiofrequency current. N Engl J Med. 1991; 324(23): 1601-11.

22. Calkins H, Kim YN, Schmaltz S, Sousa J, el -Atassi R, Leon A, et al. Electrogram criteria for identification of appropiate target sites for radiofrequency catheter ablation of accessory atrioventricular connections. Circulation. 1992; 85(2):565-73.

23.Centurión OA, Shimizu A, Isomoto S, Konoe A. Mechanism for the genesis of paroxysmal atrial fibrillation in the WolffParkinson-White syndrome: Intrinsic atrial muscle vulnerability vs electrophysiological properties of the accessory pathway. Europace. 2008; 10(3):294-302.

24.Centurión OA. Atrial fibrillation in the WolffParkinson-White syndrome. JA FB. 2011;4 (1): 22-38. 